\title{
Surfaces and Peierls Contours: 3-d Wetting and 2-d Ising Percolation
}

\author{
D. B. Abraham ${ }^{1}$ and C. M. Newman ${ }^{2, \star}$ \\ ${ }^{1}$ Department of Theoretical Chemistry, 5, South Parks Rd., Oxford OX13UB, England \\ ${ }^{2}$ Department of Mathematics and Ariz. Center for Math. Sciences, University of Arizona, \\ Tucson, AZ 85721, USA
}

\section{Dedicated to Roland Dobrushin}

\begin{abstract}
A natural model of a discrete random surface lying above a twodimensional substrate is presented and analyzed. An identification of the "level curves" of the surface with the Peierls contours of Ising spin configurations leads to an exactly solvable free energy, with logarithmically divergent specific heat. The thermodynamic critical point is shown to be a wetting transition at which the surface height diverges. This is so even though the surface has no "downward fingers" and hence no "entropic repulsion" from the substrate.
\end{abstract}

\section{Introduction}

Simple geometrical models of surfaces can be useful for understanding various aspects of interface structure and phase transitions. In this paper we analyze in detail a particular three-dimensional random surface model, first introduced in [AN] (see Sect. II for the definition). The main conclusions are:

(i) The model is directly related to the standard 2- $d$ Ising ferromagnet and thus has an exactly solvable free energy with a logarithmically divergent specific heat singularity.

(ii) The singularity corresponds to a transition from partial to complete wetting; i.e., from finite to infinite height of the surface above the substrate.

Elementary models of surfaces can be constructed on the simple cubic lattice by assigning a single-valued variable $h(x, y) \in \mathbb{Z}$ to each point $(x, y) \in \mathbb{Z}^{2}$. This variable is the height of the surface; we place a unit-sided square, or plaquette, symmetrically through the point $(x, y, h(x, y))$ parallel to the $(x, y)$ plane. We then fill in between these plaquettes with others whose normals are parallel to the $\mathbb{Z}^{2}$ plane. Such a model, with configurational energy,

$$
J \sum_{|\mathbf{r}-\mathbf{s}|=1}|h(\mathbf{r})-h(\mathbf{s})|^{p},
$$

\footnotetext{
* Research supported in part by NSF Grant No. DMS-8514834 and AFOSR Contract No. F49620-86-C0130 under the U.R.I. program
} 
usually with $p=1,2$, is termed solid-on-solid (SOS). In a seminal work, Fröhlich and Spencer [FS] proved that there is a phase transition of roughening type: this means essentially that at low temperature, $\langle|h(\mathbf{r})-h(\mathbf{0})|\rangle$ is uniformly bounded in $\mathbf{r}$, but for high temperature, $\langle|h(\mathbf{r})-h(\mathbf{0})|\rangle$ diverges as $|\mathbf{r}| \rightarrow \infty$. By introducing the additional restriction $h(\mathbf{r})-h(\mathbf{s})= \pm 1$ whenever $|\mathbf{r}-\mathbf{s}|=1$, van Beijeren [B2] introduced the $F$-SOS model - the surface ripples in the ground state, with excitations which are height changes of \pm 2 in one or other of the diagonal directions. The configurations thus constructed are isomorphic to those of the 6-vertex model with the $F$-weighting, which was solved by Lieb [L; LW] for thermodynamics: thus the $F$-SOS model has an infinite order phase transition. The low-temperature phase has been shown to have bounded fluctuations by Forrester $[\mathrm{F}]$. Only for one finite temperature is the high-temperature phase understood - there $\left\langle(h(\mathbf{r})-h(\mathbf{0}))^{2}\right\rangle \sim \log |\mathbf{r}|$ as $|\mathbf{r}| \rightarrow \infty$.

For the surface of phase-separation in the 3- $d$ Ising model, the first result is that of Dobrushin [Do] showing that at low enough temperatures the interface fluctuations are bounded. Burton et al. $[\mathrm{BCF}]$ had suggested earlier that there is a roughening transition with thermodynamic behavior exactly like the 2-d Ising model; this was based on a mean field idea, and it does not agree with the $F$-SOS results. The 2- $d$ Ising critical temperature has been proved [B1] to be a lowerbound to such a roughening transition, the existence of which is not proved since it might occur at the 3-d critical temperature and thus be trivial.

In this paper, we consider surfaces restricted to lie above a substrate which differentiates energetically between the two coexisting thermodynamic phases which are separated by the surface in question. The most obvious way to achieve this restriction is to take $h(x, y) \geqq 0$ in the above SOS model. Drawing on nonrigorous notions within the renormalization group framework, Bricmont et al. $[\mathrm{BEF}]$ have shown that the downward fluctuations in the surface are restricted by the substrate plane sufficiently strongly to drive the interface away to infinity; this is the mechanism of entropic repulsion [FF]. To produce a phase transition, one imposes a suitable binding of the surface to the substrate. This binds the surface to the substrate at low enough temperatures. At present it appears to be a mild heresy to question that the only mechanism which produces unbinding is the entropic repulsion one. Nevertheless, in the next section, we describe a random surface model with an unbinding transition which relies on a different mechanism. Thus entropic repulsion is a sufficient but not necessary condition. Our model reduces a 3-d system to an exactly solvable 2-d model, as the van Beijeren F-SOS construction does. Thus we get an exact description of the critical phenomenon associated with the transition. By calculating the step free energy in our model, we obtain additional physical evidence that our model shows simultaneous roughening and unbinding of the surface, since in the high temperature phase the step free energy vanishes. This allows deformations of the surface to proliferate. Van Beijeren has performed the same type of analysis for the F-SOS model.

As a final remark, the reason this transition is called wetting is that, if the surface separates phase $A$ below itself from phase $B$ above, at low-temperature phase $A$ is squeezed out. On going to the high temperature phase, a macroscopic slab of phase $A$ intercalates itself between $B$ and the substrate; we say $A$ wets the substrate perfectly. For further information and references, the reader may consult Dietrich [Di], van Beijeren and Nolden [BN], Weeks [W] or Abraham [A]. 


\section{The Model: Definition and Main Results}

The definition of our model given in this section is in terms of the height function $h(\mathbf{r})$ of the phase separating surface. An alternate description which focuses on the multilayers of molecules between the substrate and the surface is given in Appendix B.

We begin with the configurational energy which has two parts. The first part, consisting of a surface-tension contribution $\tau>0$ for each plaquette of the surface, is

$$
\tau \sum_{|\mathbf{r}-\mathbf{s}|=1}|h(\mathbf{r})-h(\mathbf{s})|+\tau A_{1} .
$$

The first term comes from plaquettes perpendicular to the substrate plane and the second from those parallel. $A_{1}$, the number of parallel faces, also equals the area of substrate in contact with molecules. The second term, describing a contact interaction of the molecules with the substrate, is simply

$$
-\varepsilon_{1} A_{1}=-\varepsilon_{1} \sum_{\mathrm{r}: h(\mathbf{r}) \neq 0} 1,
$$

where $\varepsilon_{1}$ is a binding energy per molecule. The total Hamiltonian is thus

$$
\mathscr{H}=\tau \sum_{|\mathbf{r}-\mathbf{s}|=1}|h(\mathbf{r})-h(\mathbf{s})|+\left(\tau-\varepsilon_{1}\right) A_{1} .
$$

To make $\mathscr{H}$ and our model well defined, we begin with a finite region $\Lambda \subset \mathbb{Z}^{2}$ of the substrate and pin the surface outside of $\Lambda$ :

(i) $h(\mathbf{r})=0$ for every $\mathbf{r}$ outside of $\Lambda$.

Of course, the surface is constrained to lie above the substrate:

(ii) $h(\mathbf{r})$ is a non-negative integer for every $\mathbf{r}$ in $\Lambda$.

There are two additional constraints to the allowed configurations. The first of these is a bound of 1 on the magnitude of the (discrete) gradient of the surface and is weaker than the $F$-SOS restriction of [B2] mentioned in the introduction:

(iii) $h(\mathbf{r})-h(\mathbf{s})=0, \pm 1$ for $|\mathbf{r}-\mathbf{s}|=1$.

The final restriction is to disallow all "downward fingers" of the surface:

(iv) For every $\mathbf{r}$ in $\Lambda$, there is some nearest-neighbor path from $\mathbf{r}$ to an $\mathbf{r}^{\prime}$ outside of $A$ along which $h$ is non-increasing.

Although the final restriction (iv) is a serious (and perhaps objectionable) one, we wish to point out that the configurations of our surface are much more general than those of the so-called wedding cake model (see, e.g., [BEF]) where there is only a single local maximum. Our surface can have many local maxima, as well as saddle points; only "hidden valleys" are ruled out. The reason for our special restrictions (iii) and (iv) is seen in the next proposition. It is a consequence of the simple fact that the contours (on the dual lattice) which separate regions with $|\Delta h|=1$ may be identified with the Peierls contours of an Ising model which separate parallel spin clusters (see also Appendix B and Fig. 1 there).

Proposition II-1. For a finite $\Lambda \subset \mathbb{Z}^{2}$, the height configurations satisfying (i)-(iv) are in one-to-one correspondence with Ising spin configurations $\left\{\sigma_{A}(\mathbf{r})= \pm 1: \mathbf{r} \in \mathbb{Z}^{2}\right\}$ in which $\sigma_{\Lambda}(\mathbf{r})=+1$ for every $\mathbf{r}$ outside $\Lambda$. The correspondence is obtained by identifying $h(\mathbf{r})$ with the minimum number of Peierls contours crossed, among all 
possible nearest neighbor paths from $\mathbf{r}$ to the complement of $\Lambda$. Under this identification, and setting $b=\varepsilon_{1}-\tau$,

$$
\mathscr{H}=-\frac{\tau}{2} \sum_{|\mathbf{r}-\mathbf{s}|=1}\left(\sigma_{\Lambda}(\mathbf{r}) \sigma_{\Lambda}(\mathbf{s})-1\right)+b \sum_{\mathbf{r} \in \Lambda}\left(\mu_{\Lambda}(\mathbf{r})-1\right),
$$

where $\mu_{\Lambda}(\mathbf{r})=1$ (otherwise 0 ) only if some path reaches from $\mathbf{r}$ to the complement of $\Lambda$ with no spin changes - i.e., only if $\mathbf{r}$ is in the same parallel spin cluster as the complement of $\Lambda$.

When $\varepsilon_{1}=\tau$, we have the 2- $d$ Ising ferromagnet in zero field with + boundary conditions and a coupling only between neighboring spins of strength $\tau / 2$. The free energy was obtained by Onsager [O]: there is a phase transition at a temperature $T=T_{c}(2)$, where

$$
\sinh \left(\tau / T_{c}(2)\right)=1
$$

with an associated logarithmic divergence of the specific heat. The notation $T_{c}(2)$ is meant to remind one that the transition in the 3- $d$ system occurs at the 2- $d$ Ising critical temperature, as originally suggested by Burton et al. [BCF] for the wetting model.

For general $\varepsilon_{1}$ and $\tau$, we have from (2.4) that the free energy $f(T, b)$, as a function of temperature $T$ and $b=\varepsilon_{1}-\tau$, is given by

$$
f(T, b)=f(T, 0)+\tilde{f}(T, b),
$$

where $f(T, 0)$ is the Onsager free energy and

$$
f(T, b)=b-T \lim _{\Lambda \rightarrow \mathbb{Z}^{2}} \frac{1}{|\Lambda|} \log \left\langle\exp \left(-\frac{b}{T} \sum_{\mathbf{r} \in \Lambda} \mu_{\Lambda}(r)\right)\right\rangle_{T} .
$$

Here the limit is in the van Hove sense, $|\Lambda|$ denotes the number of sites in $\Lambda$, and $\langle\cdot\rangle_{T}$ denotes a thermal average with respect to the Gibbs distribution of the Ising model $\left\{\sigma_{\Lambda}(\mathbf{r})\right\}$ at temperature $T$ (with $b$ set to zero in $\mathscr{H}$ ). The next proposition shows that the thermodynamic singularity at $T=T_{c}(2)$ is unchanged for $\varepsilon_{1}>\tau$; the proof may be found in Sect. IV below. The simple formula obtained for the free energy difference $\tilde{f}$ is closely related to the fact (see also Theorem II-4 below) that the surface configurations for $b=0$ and $b>0$ essentially differ only in the presence of an extra monolayer of molecules sticking to the substrate when $b>0$.

Theorem II-2. For $0<T<\infty$ and $b \equiv \varepsilon_{1}-\tau \geqq 0$, the free energy satisfies

$$
f(T, b)=f(T, 0)+b \text {. }
$$

The next theorem identifies the $b \geqq 0, T \geqq T_{c}(2)$ phase of the random surface as a wet phase in which the surface diverges as $\Lambda \rightarrow \mathbb{Z}^{2}$ (through any sequence of finite regions which eventually cover all of $\left.\mathbb{Z}^{2}\right)$. We denote by $\left\{h_{\Lambda}(\mathbf{r}): \mathbf{r} \in \mathbb{Z}^{2}\right\}$ the random heights given by our model with Gibbs distribution proportional to $\exp (-\mathscr{H} / T)$; the $T$ and $b$ dependence is suppressed in this notation. The $b=0$ results are derived in Sect. III and extended to $b>0$ in Sect. IV. The theorem also gives, from Sect. V, a partial result on the height divergence rate, valid for large $T$. 
Theorem II-3. For $T \geqq T_{c}(2)$ and $b \geqq 0$, the height of the surface at the origin $h_{A}(\mathbf{0})$ diverges as $\Lambda \rightarrow \mathbb{Z}^{2}$, in the sense that there is some choice of constants $K_{\Lambda}$ (depending on $T$, but not b) with $K_{\Lambda} \rightarrow \infty$ as $\Lambda \rightarrow \mathbb{Z}^{2}$, such that

$$
\operatorname{Pr}\left(h_{\Lambda}(\mathbf{0}) \geqq K_{\Lambda}\right) \rightarrow 1 \quad \text { as } \quad \Lambda \rightarrow \mathbb{Z}^{2} .
$$

For $T$ sufficiently large, $K_{\Lambda}$ may be taken as

$$
K_{\Lambda}=\gamma^{\prime} \bar{R}(\Lambda) / \log (\bar{R}(\Lambda)),
$$

where $\bar{R}(\Lambda)$ is the distance from $\mathbf{0}$ to the boundary of $\Lambda$.

Remark. We believe that the actual height divergence is linear in $\bar{R}(\Lambda)$ and that this is valid for all $T>T_{c}(2)$. At $T=T_{c}(2)$, the divergence should be slower, perhaps like a fractional power of $\bar{R}$.

The next theorem shows that for $b \leqq 0$ and $T<T_{c}(2)$, the substrate is only partially wet in the sense that the wet regions (droplets) remain finite in both height and lateral extent as $\Lambda \rightarrow \mathbb{Z}^{2}$. This remains valid for $b>0$ except for the presence of a wet monolayer. For $b \geqq 0$ the mean height diverges as $T \rightarrow T_{c}(2)$ from below, but no faster than $\left(T_{c}-T\right)^{-1 / 8}$. The $b=0$ results of the theorem are derived in Sect. III, the $b \neq 0$ results in Sect. IV. We define the droplet of the origin $\mathscr{D}_{A}$ as the connected cluster of sites with $h_{\Lambda}(\mathbf{r}) \geqq 1$ which contains the origin [if $h_{A}(\mathbf{0})=0$, then $\mathscr{D}_{\Lambda}$ is the empty set].

Theorem II-4. For $b \leqq 0$ and $T<T_{c}(2)$, the droplet $\mathscr{D}_{\Lambda}$ remains finite as $\Lambda \rightarrow \mathbb{Z}^{2}$ in the sense that

$$
\lim _{k \rightarrow \infty}\left[\sup _{\Lambda} \operatorname{Pr}\left(\left|\mathscr{D}_{\Lambda}\right| \geqq k\right)\right]=0 .
$$

For $T<T_{c}(2)$ and any $b$, the height order parameter,

$$
\theta(T, b) \equiv \sup _{\Lambda} E\left(h_{\Lambda}(\mathbf{0})\right)
$$

(which equals $\lim _{\Lambda} E\left(h_{\Lambda}(\mathbf{0})\right)$ for $\left.b \geqq 0\right)$ is finite and satisfies

$$
\begin{array}{cc}
\theta(T, b) \leqq \theta(T, 0), & \text { for } \quad b<0, \\
\theta(T, b)=1+\theta(T, 0), & \text { for } b>0, \\
\theta(T, b) \leqq \operatorname{const}\left(T_{c}(2)-T\right)^{-1 / 8}, & \text { for } \quad b=0 .
\end{array}
$$

Finally, for $b \geqq 0, \theta(T, b) \rightarrow+\infty$ as $T \rightarrow T_{c}(2)$ from below.

Remark. A slightly stronger result than (2.11) can be obtained in which the droplet $\mathscr{D}_{A}$ (a connected cluster) is replaced by a larger $*$-connected cluster. This can be based on the percolation results of [R;GKR].

We conclude from our analysis so far, that for $\varepsilon_{1} \geqq \tau$ we have a wetting transition at $T=T_{c}(2)$. The free energy per unit substrate area is that obtained by Onsager [O]. The fact that this is singular as $T \rightarrow T_{c}(2)+$ in the present context is curious. We believe that as $T$ increases through $T_{c}(2)$ there is a simultaneous roughening transition; this is supported, but certainly not proved by a calculation of the step free energy given next for $\varepsilon_{1}=\tau$. 
A moment's reflection shows that we can introduce a unit height step crossing the lattice at an angle $\theta$ between points $A$ and $B$ say on the boundary of an $N \times M$ strip by imposing Dobrushin +- boundary conditions at $A$ and $B$ [Do] giving a modified partition function $Z_{N M}^{+-}$instead of the $Z_{N M}$ obtained with + boundary conditions. Let the Euclidean length from $A$ to $B$ be (approximately) $N / \cos \theta$.

Definition. The step free energy at angle $\theta$ is

$$
\sigma(\theta)=-\lim _{N \rightarrow \infty} \frac{|\cos \theta|}{N} \lim _{M \rightarrow \infty} \log \frac{Z_{N M}^{+}}{Z_{N M}} .
$$

Remark. This is exactly the $2-d$ Ising model surface tension at angle $\theta$ which is already known [AM; AR]; this calculation is actually for $T \leqq T_{c}(2)$. The result for $T>T_{c}(2)$ is most easily got by noting that the dual of $Z_{N}^{+-} / Z_{N}$ [the inner limit of (2.14)] is a pair correlation function in a strip, from which the vanishing of $\sigma(\theta)$ in that case follows readily.

Theorem II-5. For all $T>T_{c}(2), \sigma(\theta)=0$.

Remark. This is an intuitive condition for roughness of the surface. A more appealing result, which we have not established, would be to show

$$
\lim _{|\mathbf{r}-\mathbf{s}| \rightarrow \infty} \liminf _{\boldsymbol{\Lambda} \rightarrow \mathbb{Z}^{2}} E\left(\left|h_{\Lambda}(\mathbf{r})-h_{\Lambda}(\mathbf{s})\right|\right)=\infty .
$$

\section{Results when $\varepsilon_{1}=\tau$}

In this section we set $b \equiv \varepsilon_{1}-\tau=0$. As explained in Proposition II-1 and in Appendix B, the flat parts of the phase-separating surface are represented in the planar Ising model equivalence by connected clusters of parallel spins and the height $h_{A}(\mathbf{r})$ is the minimum number of Peierls contours crossed, over all nearest neighbor paths from $\mathbf{r}$ to the boundary of $\Lambda$. We proceed to develop the relationship of percolative ideas to this random surface model.

The key theorem on clusters in the planar Ising model is due to Coniglio et al. [CNPR]:

Theorem III-1 [CNPR]. Consider the standard 2-d Ising ferromagnet with zero external field at temperature $T$. If $T \geqq T_{c}(2)$, then the (unique) infinite volume Gibbs state has, with probability one, no infinite connected cluster of parallel spins. If $T<T_{c}(2)$, then the plus boundary condition infinite volume Gibbs state has, with probability one, exactly one infinite connected cluster of plus spins; further, the connected components of the complement of that infinite cluster (consisting of both minus spin sites and those plus spin sites not part of the infinite plus cluster) are all finite, with probability one.

This result of Coniglio et al. yields as a corollary the next theorem, which demonstrates that the thermodynamic singularity at $T_{c}(2)$ corresponds to a wetting transition in which the surface height remains finite (in the infinite volume limit) for $T<T_{c}(2)$ but diverges for $T \geqq T_{c}(2)$. This can readily be seen to follow from the Coniglio et al. percolation analysis, if we disregard, for the time being, some technical issues associated with the infinite volume limit: In the infinite system, 
define the surface height $H(\mathbf{r})$ at a point $\mathbf{r}=(x, y) \in \mathbb{Z}^{2}$ as the minimum number of Peierls contours that must be crossed for a nearest neighbor path from $\mathbf{r}$ to reach infinity. When $T \geqq T_{c}(2)$ all parallel spin clusters are finite and hence infinitely many Peierls contours must be crossed; thus $H(\mathbf{r})=+\infty$ (with probability one) for $T \geqq T_{c}(2)$. When $T<T_{c}(2)$ only finitely many contours need be crossed to reach the infinite plus cluster and then no more crossings are needed; thus $H(\mathbf{r})<\infty$ (with probability one) for $T<T_{c}(2)$. The next theorem takes care of the infinite volume limit. The random variables $\left\{h_{\Lambda}(\mathbf{r}): \mathbf{r} \in \mathbb{Z}^{2}\right\}$ appearing next are the heights obtained from the Ising model $\left\{\sigma_{\Lambda}(\mathbf{r})\right\}$ defined with plus boundary conditions outside the finite region $\Lambda \subset \mathbb{Z}^{2}$ (see Appendix A); $h_{\Lambda}(\mathbf{r})=0$ for $\mathbf{r}$ outside $\Lambda$.

Theorem III-2. For $T \geqq T_{c}(2), E\left(h_{\Lambda}(\mathbf{r})\right) \rightarrow+\infty$ for each $\mathbf{r}$ as $\Lambda \rightarrow \mathbb{Z}^{2}$ because $h_{\Lambda}(\mathbf{r})$ $\rightarrow+\infty$ in probability; i.e., for any finite $k$

$$
\operatorname{Pr}\left(h_{\Lambda}(\mathbf{r}) \geqq k\right) \rightarrow 1 \quad \text { as } \quad \Lambda \rightarrow \mathbb{Z}^{2} \text {. }
$$

For $T<T_{c}(2)$, the $h_{\Lambda}(\mathbf{r})$ 's tend to the finite $H(\mathbf{r})$ 's as $\Lambda \rightarrow \mathbb{Z}^{2}$ in the sense that for any $m$ and $\mathbf{r}_{1}, \ldots, \mathbf{r}_{m}$,

$$
\lim _{\Lambda \rightarrow \mathbb{Z}^{2}} E\left(f\left(h_{\Lambda}\left(\mathbf{r}_{1}\right), \ldots, h_{\Lambda}\left(\mathbf{r}_{m}\right)\right)\right)=E\left(f\left(H\left(\mathbf{r}_{1}\right), \ldots, H\left(\mathbf{r}_{m}\right)\right)\right)
$$

for any function $f$ which is either bounded or else is non-decreasing in each coordinate.

Proof. It suffices to show (see Proposition A3 of Appendix A) that as $\Lambda \rightarrow \mathbb{Z}^{2}$,

$$
G_{\Lambda} \equiv \operatorname{Pr}\left(h_{\Lambda}\left(\mathbf{r}_{1}\right) \geqq k_{1}, \ldots, h_{\Lambda}\left(\mathbf{r}_{m}\right) \geqq k_{m}\right) \rightarrow G \equiv \operatorname{Pr}\left(H(\mathbf{r}) \geqq k_{1}, \ldots, H\left(\mathbf{r}_{m}\right) \geqq k_{m}\right) .
$$

Denote by $H_{N}(\mathbf{r})$ the minimum number of Peierls contours that must be crossed in the infinite volume system to walk from $\mathbf{r}$ to the boundary of $\mathbf{r}+\Lambda_{N N}$ and by $h_{\Lambda, N}(\mathbf{r})$ the corresponding quantity in the finite region $\Lambda$ system; let $G_{{ }_{, N}}$ and $G_{\Lambda, N}$ denote the corresponding finite $N$ approximations to $G$ and $G_{\Lambda}$. Since as $N \rightarrow \infty, H_{N}(\mathbf{r})$ and $h_{\Lambda, N}(\mathbf{r})$ increase to $H(\mathbf{r})$ and $h_{\Lambda}(\mathbf{r})$, it follows that $G_{, N}$ and $G_{\Lambda, N}$ increase to $G$ and $G_{\Lambda}$. On the other hand, $G_{\Lambda, N} \rightarrow G_{, N}$ as $\Lambda \rightarrow \mathbb{Z}^{2}$ because they involve the probabilities of events depending only on a fixed finite set of spin variables (those in $\bigcup_{i}\left(\mathbf{r}_{i}+\Lambda_{N N}\right)$ ). Thus

$$
\liminf _{\Lambda \rightarrow \mathbb{Z}_{2}} G_{\Lambda} \geqq \lim _{N \rightarrow \infty}\left(\lim _{\Lambda \rightarrow \mathbb{Z}^{2}} G_{\Lambda, N}\right)=\lim _{N \rightarrow \infty}\left(G_{, N}\right)=G .
$$

This already proves the $T \geqq T_{c}(2)$ result, (3.1). It remains to show that $G_{\Lambda} \leqq G$ for $T<T_{c}(2)$. To do this we need to extend the representation (A.5) and inequality (A.6) of Appendix A to the case where $\Lambda$ is replaced by $\mathbb{Z}^{2}$. Toward this end, let us define $\mu(\mathbf{r})$ to be 1 (or 0 ) when $H(\mathbf{r})$ is 0 (or nonzero) and for the given $\mathbf{r}_{1}, \ldots, \mathbf{r}_{m}$, let us define the random subset $L$ of $\mathbb{Z}^{d}$ as those sites $\mathbf{r}$ with $\mu(\mathbf{r})=0$ which are not the nearest neighbor of some $\mathbf{r}^{\prime}$ with $\mu\left(\mathbf{r}^{\prime}\right)=0$ and which are connected to some $\mathbf{r}_{i}$ by a walk passing over only sites with $\mu=0$. A key consequence of the latter part of Theorem II-1 is that for $T<T_{c}(2), L$ is finite with probability one. We then have, as an extension of (A.5),

$$
\left\{H\left(\mathbf{r}_{i}\right): i=1, \ldots, m\right\} \cong\left\{\left(1-\mu\left(\mathbf{r}_{i}\right)\right)\left(1+h_{L}\left(\mathbf{r}_{i}\right)\right): i=1, \ldots, m\right\},
$$


where $\cong$ denotes equidistribution. By an argument substantially the same as used for Proposition A1 and by Proposition A1 itself we obtain the desired extension of (A.6).

For $T<T_{c}(2)$, Theorem II-2 states that the interface surface height remains finite. Theorem II-1 further implies that the "dry" region, $\left\{\mathbf{r} \in \mathbb{Z}^{2}: H(\mathbf{r})=0\right\}$, percolates while the "wet" region, $\left\{r \in \mathbb{Z}^{2}: H(\mathbf{r}) \geqq 1\right\}$, does not. Thus, to mix metaphors, the wet region consists entirely of finite droplets within a dry desert; this combined with Proposition A1 of Appendix A yields (2.11) for $b=0$. Some explicit estimates of the height of these droplets are possible in terms of the Ising model spontaneous magnetization [Y; BGJS; AM],

$$
m^{*}(T)=\left[1-1 / \sinh ^{4}(\tau / T)\right]^{1 / 8} .
$$

Theorem III-3. For $T<T_{c}(2)$ and any finite $\Lambda$ and positive integer $k$,

$$
\begin{gathered}
\operatorname{Pr}\left(h_{\Lambda}(\mathbf{r}) \geqq k\right) \leqq \operatorname{Pr}(H(\mathbf{r}) \geqq k) \leqq\left(1-m^{*}\right)^{k}, \\
E\left(h_{\Lambda}(\mathbf{r})\right) \leqq E(H(\mathbf{r})) \leqq\left(1-m^{*}\right) / m^{*} .
\end{gathered}
$$

Furthermore, for any $k$,

$$
\lim _{T \rightarrow T_{c}(2)-} \operatorname{Pr}(H(\mathbf{r}) \geqq k)=1,
$$

and hence $E(H(\mathbf{r}))$ diverges as $T$ tends to $T_{c}(2)$ from below; i.e., the surface height diverges continuously at $T_{c}(2)$.

Proof. Let us define

$$
\bar{L}_{\Lambda}=\left\{\mathbf{r}: h_{\Lambda}(\mathbf{r})>0\right\}=\text { the complement of the plus-cluster of } \mathbb{Z}^{d} \backslash \Lambda \text {. }
$$

The spins in $\bar{L}_{\Lambda}$ have minus boundary conditions on the "inside" boundary of $\bar{L}_{\Lambda}$. Let us denote by $\AA$ the interior of $A \cong \mathbb{Z}^{d}$, i.e., those $\mathbf{r}$ in $A$ which are not neighbors of some $\mathbf{r}^{\prime}$ outside of $A$. Then for $k \geqq 1$ [compare Eq. (A.5) of the appendix]

$$
\operatorname{Pr}\left(h_{\Lambda}(\mathbf{r}) \geqq k\right)=\sum_{A: \mathbf{r} \in A} \operatorname{Pr}\left(\bar{L}_{A}=A\right) \operatorname{Pr}\left(h_{\mathcal{A}}(\mathbf{r}) \geqq k-1\right) .
$$

Now by the monotonicity in $\Lambda^{\prime}$ of $h_{\Lambda^{\prime}}$ (Proposition A2),

$$
\operatorname{Pr}\left(h_{A}(\mathbf{r}) \geqq k-1\right) \leqq \operatorname{Pr}\left(h_{\Lambda}(\mathbf{r}) \geqq k-1\right),
$$

so that

$$
\begin{aligned}
\operatorname{Pr}\left(h_{\Lambda}(\mathbf{r}) \geqq k\right) & \leqq \operatorname{Pr}\left(h_{\Lambda}(\mathbf{r}) \geqq k-1\right) \cdot \operatorname{Pr}\left(\mathbf{r} \in \bar{L}_{\Lambda}\right) \\
& =\operatorname{Pr}\left(h_{\Lambda}(\mathbf{r}) \geqq k-1\right) \cdot \operatorname{Pr}\left(h_{\Lambda}(\mathbf{r}) \geqq 1\right) \\
& \leqq \operatorname{Pr}\left(h_{\Lambda}(\mathbf{r}) \geqq k-1\right) \cdot \operatorname{Pr}(H(\mathbf{r}) \geqq 1),
\end{aligned}
$$

where the last inequality used Proposition A2 and Theorem III-2. Now Coniglio et al. [CNPR, Theorem 1] proved that $\operatorname{Pr}(H(\mathbf{r})=0) \geqq m^{*}$, so that (3.12) yields

$$
\operatorname{Pr}\left(h_{\Lambda}(\mathbf{r}) \geqq k\right) \leqq\left(1-m^{*}\right) \operatorname{Pr}\left(h_{\Lambda}(\mathbf{r}) \geqq k-1\right)
$$

which (again using Proposition A2 and Theorem III-2) leads to (3.7) and thence to (3.8). 
To obtain (3.9), we note from Proposition A2 and Theorem III-2, that $\operatorname{Pr}(H(\mathbf{r}) \geqq k)$ as a function of $T$ is the increasing limit of the continuous functions $\operatorname{Pr}\left(h_{\Lambda}(\mathbf{r}) \geqq k\right)$ as $\Lambda \rightarrow \mathbb{Z}^{2}$ through some increasing sequence of finite regions $\Lambda_{n}$. Hence $\operatorname{Pr}(H(\mathbf{r}) \geqq k)$ is lower semicontinuous. Since it is bounded above by 1 and equals 1 at $T=T_{c}(2),(3.9)$ follows by standard arguments.

Remark. Somewhat stronger results than (3.7)-(3.8) are given in Proposition A4 of Appendix A.

\section{Results for General $\varepsilon_{1}, \tau$}

We now relax the condition $\varepsilon_{1}=\tau$ necessary to reduce the problem to the $2-d$ Ising one with zero field. When $b \equiv \varepsilon_{1}-\tau \neq 0$, we have [see (2.4)], in addition to the usual Ising model Boltzmann weight, the additional factor,

$$
e^{-\beta b \sum \mu_{\Lambda}(\mathbf{r})}
$$

up to a configuration-independent factor. For a given inverse temperature $\beta=1 / T$, we denote by $\left\{h_{\Lambda, b}(\mathbf{r}): \mathbf{r} \in \mathbb{Z}^{2}\right\}$ the corresponding height random variables; their expectations are related to those of $\left\{h_{\Lambda, 0}(\mathbf{r})\right\} \equiv\left\{h_{\Lambda}(\mathbf{r})\right\}$ by:

$$
E\left(f\left(\left\{h_{\Lambda, b}(\mathbf{r})\right\}\right)\right)=\frac{E\left(f\left(\left\{h_{\Lambda}(\mathbf{r})\right\}\right) e^{-\beta \Sigma_{\Lambda} \mu_{\Lambda}(\mathbf{r})}\right)}{E\left(e^{-\beta \Sigma_{\Lambda} \mu_{\Lambda}(\mathbf{r})}\right)}=\frac{\left\langle f e^{-\beta \Sigma_{\Lambda} \mu_{\Lambda}(\mathbf{r})}\right\rangle_{T}}{\left\langle e^{-\beta \Sigma_{\Lambda} \mu_{\Lambda}(\mathbf{r})}\right\rangle_{T}}
$$

where $\langle\cdot\rangle_{T}$ is the usual Ising model thermal average at temperature $T$ for $\left\{\sigma_{\Lambda}(\mathbf{r})\right\}$. For later use, we will also define $\mu_{\Lambda, b}(\mathbf{r})$ to be the indicator variable for $h_{\Lambda, b}(\mathbf{r})=0$ [so that $\left.\mu_{\Lambda, 0}(\mathbf{r})=\mu_{\Lambda}(\mathbf{r})\right]$.

This suggests using the FKG property of Appendix A where possible. In fact this leads to the following two propositions, which, together with the results previously derived for $b=0$, yield most of Theorems II-2 and II-3 - in particular (2.9), (2.11) and the $b \leqq 0$ part of (2.13). The symbol $\succ$ in the propositions denotes stochastic domination (see (A.13)).

Proposition IV-1. For $b>0$ (resp. $b<0)$

$$
\left\{h_{\Lambda, b}(\mathbf{r})\right\} \succ(\text { resp. } \prec)\left\{h_{\Lambda, 0}(\mathbf{r})\right\} \equiv\left\{h_{\Lambda}(\mathbf{r})\right\} .
$$

Proof. Use (4.2), the fact that the $\left\{\mu_{\Lambda}(\mathbf{r})\right\}$ are decreasing functions of the $\left\{h_{\Lambda}(\mathbf{r})\right\}$ and that $\left\{h_{\Lambda}(\mathbf{r})\right\}$ has the FKG property (Proposition A2). Note that $\exp \left(-\beta b \sum \mu(\mathbf{r})\right)$ is increasing (resp. decreasing) in the $\left\{h_{A}(\mathbf{r})\right\}$ for $b>0$ (resp. $b<0$ ), from which the result follows by a standard argument.

Proposition IV-2. For $b<0$

$$
\left\{h_{\Lambda, b}(\mathbf{r}): \mathbf{r} \in \Lambda\right\} \prec\left\{1+h_{\Lambda, 0}(\mathbf{r}): \mathbf{r} \in \Lambda\right\} .
$$

Proof. The generalization of (A.5) to $b \neq 0$ is

$$
\left\{h_{\Lambda, b}(\mathbf{r})\right\} \cong\left\{\left(1-\mu_{\Lambda, b}(\mathbf{r})\right)\left(1+h_{L_{\Lambda}, 0}(\mathbf{r})\right)\right\}
$$


because inside $L_{A}$ there is no $b$ dependence since the surface is entirely lifted above the substrate plane. By Proposition A1, we get

$$
\left\{h_{\Lambda, b}(\mathbf{r})\right\} \prec\left\{\left(1-\mu_{\Lambda, b}(\mathbf{r})\right)\left(1+h_{\Lambda, 0}(\mathbf{r})\right)\right\} \prec\left\{1+h_{\Lambda, 0}(\mathbf{r})\right\},
$$

(where the $\mu_{\Lambda, b}$ and $h_{\Lambda, 0}$ sets of variables in the middle expression are treated as independent).

Remark. For $b>0$ and $T<T_{c}(2)$ we shall prove below that $\left\{h_{\Lambda, b}(\mathbf{r})\right\} \rightarrow\left\{1+h_{\Lambda, 0}(\mathbf{r})\right\}$ as $\Lambda \rightarrow \mathbb{Z}^{2}$.

We now turn our attention to the free energy as defined by (2.6)-(2.7). The next proposition gives half of Theorem II-2. We continue to denote $1 / T$ by $\beta$.

Proposition IV-3. For $T \geqq T_{c}(2)$ and $b \geqq 0, \tilde{f}(T, b)=b$.

Proof. By Jensen's inequality,

$$
1 \geqq\left\langle\exp \left(-\beta b \sum \mu_{\Lambda}(\mathbf{r})\right)\right\rangle_{T} \geqq \exp \left(-\beta b \sum_{\mathbf{r}}\left\langle\mu_{\Lambda}(\mathbf{r})\right\rangle_{T}\right) .
$$

Thus it suffices to show that

$$
\frac{1}{|\Lambda|} \sum_{\mathbf{r} \in \Lambda}\left\langle\mu_{\Lambda}(\mathbf{r})\right\rangle_{T} \rightarrow 0 \quad \text { as } \quad \Lambda \rightarrow \mathbb{Z}^{2}
$$

(in the van Hove sense). But, for fixed $\mathbf{r},\left\langle\mu_{\Lambda}(\mathbf{r})\right\rangle_{T}=\operatorname{Pr}\left(h_{\Lambda}(\mathbf{r})=0\right)$ tends monotonically to zero as $\Lambda \rightarrow \mathbb{Z}^{2}$ by Proposition A1 and Theorem III-2, from which the result follows by the definition of van Hove convergence and a standard argument.

The motivation behind the next theorem is the fact that if $b>0$, then for a large enough system the ground state has a monolayer. We then have

Theorem IV-4. For any $b>0$,

$$
\operatorname{Pr}\left\{h_{\Lambda, b}(\mathbf{0})=0\right\}=E\left(\mu_{\Lambda, b}(\mathbf{0})\right) \rightarrow 0 \quad \text { as } \quad \Lambda \rightarrow \mathbb{Z}^{2} .
$$

Also

$$
\frac{1}{|\Lambda|} \sum_{\mathbf{r} \in \Lambda} E\left(\mu_{\Lambda, b}(\mathbf{r})\right) \rightarrow 0 \quad \text { as } \quad \Lambda \rightarrow \mathbb{Z}^{2}
$$

(in the van Hove sense).

The proof will be given after the two following corollaries which show why Theorem IV-4 is useful in determining the phase diagram. These two corollaries complete the proofs of Theorems II-2 and II-4.

Corollary IV-5. For $b>0$ and $T<T_{c}(2)$,

$$
\left\{h_{\Lambda, b}(\mathbf{r}): \mathbf{r} \in \mathbb{Z}^{2}\right\} \rightarrow\left\{1+H(\mathbf{r}): \mathbf{r} \in \mathbb{Z}^{2}\right\} \quad \text { as } \quad \Lambda \rightarrow \mathbb{Z}^{2}
$$

in the sense of convergence of the expectation of bounded functions of finitely many variables.

Proof of Corollary $I V-5$. By conditioning on $L_{\Lambda, b}$ in (4.4), it suffices to prove that $L_{\Lambda, b} \rightarrow \mathbb{Z}^{2}$ as $\Lambda \rightarrow \mathbb{Z}^{2}$. From the definition of $L_{\Lambda, b}$, we have to prove that $\left\{\mathbf{r}: h_{\Lambda, b}(\mathbf{r})\right.$ 
$\neq 0\} \rightarrow \mathbb{Z}^{2}$; this is equivalent to proving $\operatorname{Pr}\left\{h_{\Lambda, b}(\mathbf{r})=0\right\} \rightarrow 0$ as $\Lambda \rightarrow \mathbb{Z}^{2}$ which is precisely the content of the first part of Theorem IV-4.

Corollary IV-6. For $b>0$ and $T<T_{c}(2), \tilde{f}(T, b)=b$.

Proof of Corollary IV-6. Choose $0<\varepsilon<b$; then

$$
1 \geqq\left\langle e^{-\beta b \Sigma_{\Lambda} \mu_{\Lambda}(\mathbf{r})}\right\rangle_{T}=\left\langle e^{-\beta \varepsilon \Sigma_{\Lambda} \mu_{\Lambda}(\mathbf{r})}\right\rangle_{T} \cdot E\left(e^{-\beta(b-\varepsilon) \Sigma_{\Lambda} \mu_{\Lambda, \varepsilon}(\mathbf{r})}\right) \geqq e^{-\beta \varepsilon|\Lambda|} e^{-\beta(b-\varepsilon) E\left(\sum_{\Lambda} \mu_{\Lambda, \varepsilon}(\mathbf{r})\right)}
$$

by Jensen's inequality. We can now apply the second part of Theorem IV-4 to see that $0 \leqq \widetilde{f}(T, b)-b \leqq \varepsilon$ from which the desired result follows by letting $\varepsilon$ tend to zero.

Proof of Theorem $I V-4$. For a given finite $\Lambda, \beta=1 / T$, and $b>0$, we denote by $\varrho_{b}$ the Gibbs distribution (with plus boundary conditions outside $\Lambda$ ) on the spin configurations $\{\sigma(\mathbf{r}): \mathbf{r} \in \Lambda\}$ with density $e^{-\beta \mathscr{H}_{b}}, \mathscr{H}_{b}$ given by (2.4). We denote by $\mu(\mathbf{r})$ the function of the spin configurations which assigns one (otherwise 0 ) if $\mathbf{r}$ is in the plus spin cluster of the complement of $\Lambda(\mu(\mathbf{r})$ is a realization of the random variable $\left.\mu_{\Lambda, b}(\mathbf{r})\right)$. To prove (4.6), we will give a precise version of the argument that the event $\left\{|\Lambda|^{-1} \sum_{\Lambda} \mu(\mathbf{r}) \geqq \varepsilon\right\}$ has small $\varrho_{b}$ measure since this event can be avoided by flipping a moderate number of spins.

We define a spin flip mapping $\phi_{\tilde{\Lambda}}$ for each $\tilde{\Lambda} \subset \Lambda$, which only alters the spins in $\partial \tilde{\Lambda}=\left\{\mathbf{r} \in \Lambda: \mathbf{r}\right.$ is a nearest neighbor of some $\left.\mathbf{r}^{\prime} \notin \Lambda\right\}$ as $\phi_{\tilde{\Lambda}}:\{\sigma(\mathbf{r})\} \mapsto\left\{\sigma^{\prime}(\mathbf{r})\right\}$, where

$$
\sigma^{\prime}(\mathbf{r})= \begin{cases}-1, & \text { if } \mathbf{r} \in \partial \tilde{\Lambda} \text { and } \mu(\mathbf{r})=1 \\ \sigma(\mathbf{r}), & \text { otherwise }\end{cases}
$$

We remark that:

(i) $\phi_{\tilde{\Lambda}}$ is not 1 -to-1, but is at most $2^{|\partial \tilde{\Lambda}|}-$ to-1.

(ii) $\left\{\sigma^{\prime}(\mathbf{r})\right\}$ has $\mu^{\prime}(\mathbf{r})=0$ for every $\mathbf{r} \in \tilde{\Lambda}$.

(iii) If $\mu^{\prime}(\mathbf{r})=1$, then $\mu(\mathbf{r})=1$.

It follows from (ii)-(iii) that

$$
\mathscr{H}_{b}\left(\left\{\sigma^{\prime}(\mathbf{r})\right\}\right) \leqq \mathscr{H}_{b}(\{\sigma(\mathbf{r})\})+4 \tau|\partial \tilde{\Lambda}|-b \sum_{\mathbf{r} \in \boldsymbol{\Lambda}} \mu(\mathbf{r}) .
$$

Combining this with (i), we see that for any event $A$,

$$
\varrho_{b}(A) \leqq 2^{|\partial \tilde{\Lambda}|}\left(\max _{\{\sigma(\mathbf{r})\} \in A}\left[e^{-\beta b \sum_{\Lambda} \mu(\mathbf{r})}\right]\right) e^{4 \beta \tau|\partial \tilde{\Lambda}|} \varrho_{b}\left(\phi_{\tilde{\Lambda}}(A)\right),
$$

where $\phi_{\tilde{\Lambda}}(A)$ denotes the set of transformed spin configurations from $A$. Using (4.9) and $\varrho_{b}\left(\phi_{\tilde{\Lambda}}(A)\right) \leqq 1$, we take $\tilde{\Lambda}=\Lambda$ and $A$ the event that $|\Lambda|^{-1} \sum_{\Lambda} \mu(\mathbf{r}) \geqq \varepsilon$ to obtain

$$
\operatorname{Pr}\left(|\Lambda|^{-1} \sum_{\mathbf{r} \in \Lambda} \mu_{\Lambda, b}(\mathbf{r}) \geqq \varepsilon\right) \geqq 2^{|\partial \Lambda|} e^{-\beta b \varepsilon|\Lambda|} e^{4 \beta \tau|\partial \Lambda|} .
$$

This bound tends to zero for any fixed $\varepsilon$, since $|\partial \Lambda| /|\Lambda| \rightarrow 0$ as $\Lambda \rightarrow \mathbb{Z}^{2}$ in the van Hove sense, which implies the second part of Theorem IV-4, i.e., (4.6). 
For the first part, a different mapping is needed. We define, for each $\kappa$ such that $C_{\kappa} \subset \Lambda$, where $C_{\kappa}=\{(x, y):|x| \leqq \kappa,|y| \leqq \kappa\}$, the mapping $\psi_{\kappa}:\{\sigma(\mathbf{r})\} \mapsto\left\{\hat{\sigma}^{\kappa}(\mathbf{r})\right\}$, where

$$
\hat{\sigma}^{\kappa}(\mathbf{r})= \begin{cases}-2, & \text { if } \mathbf{r} \in\left[\partial C_{\kappa}\right]^{\mathbf{0}} \\ \sigma(\mathbf{r}), & \text { otherwise, }\end{cases}
$$

where $\left[\partial C_{\kappa}\right]^{\mathbf{0}}=\left\{\mathbf{r}: \mathbf{r} \in \partial C_{\kappa}\right.$ and there is a path of + spins within $C_{\kappa} \backslash \partial C_{\kappa}$ between $\mathbf{0}$ and some neighbor of $\mathbf{r}\}$. We remark that:

(i) If every configuration in $\tilde{A}$ has $\left|\left[\partial C_{\kappa}\right]^{0}\right| \leqq N$, then on $\tilde{A}$, $\psi_{\kappa}$ is at most $2^{N}$-to-1.

(ii) $\left\{\hat{\sigma}^{\kappa}(\mathbf{r})\right\}$ has $\hat{\mu}^{\kappa}(\mathbf{0})=0$.

(iii) If $\hat{\mu}^{\kappa}(\mathbf{r})=1$, then $\mu(\mathbf{r})=1$.

The analogue of (4.9) is as follows, where we let $\left[C_{\kappa}\right]^{0}=\left\{\mathbf{r}^{\prime} \in C_{\kappa} \backslash \partial C_{\kappa}\right.$ : there is a path of + spins within $C_{\kappa} \backslash \partial C_{\kappa}$ connecting $\mathbf{0}$ to $\left.\mathbf{r}\right\}$ : if every configuration in $A$ has $\mu(\mathbf{0})=1$, then

$$
\begin{aligned}
\varrho_{b}(A) & \leqq \max _{\{\sigma(\mathbf{r}) \in A}\left(2^{\left|\left[\partial C_{\kappa}\right]^{0}\right|} e^{-\beta b\left|\left[C_{\kappa}\right]^{0}\right|} e^{4 \beta \tau \tau\left|\left[\partial C_{\kappa}\right]^{0}\right|}\right) \cdot \varrho_{b}\left(\psi_{\kappa}(A)\right) \\
& \leqq e^{-\beta b \kappa} \max _{\{\sigma(\mathbf{r})\} \in A}\left\{\left(2 e^{4 \beta \tau}\right)^{\left|\left[\partial C_{\kappa}\right]^{\natural}\right|}\right\} .
\end{aligned}
$$

Thus

$$
\varrho_{b}\left(\left\{\mu(\mathbf{0})=1 \text { and }\left|\left[\partial C_{\kappa}\right]^{\mathbf{0}}\right| \leqq N\right\}\right) \leqq\left(2 e^{4 \beta \tau}\right)^{N} e^{-\beta b \kappa}
$$

and

$$
\begin{aligned}
\varrho_{b}(\{\mu(\mathbf{0}) & \left.\left.=1 \text { and }\left|\left[\partial C_{\kappa}\right]^{0}\right| \leqq N \text { for some } \kappa \geqq \kappa_{0}\right\}\right) \\
& \leqq\left(2 e^{4 \beta \tau}\right)^{N} e^{-\beta b \kappa_{0}} /\left(1-e^{-\beta b}\right) .
\end{aligned}
$$

To show that $\varrho_{b}(\{\mu(\mathbf{0})=1\}) \rightarrow 0$, we need another bound to combine with (4.12). Let $\tilde{\kappa}$ be the largest $\kappa$ such that $C_{\kappa} \subset \Lambda$. Then, applying $\phi_{C_{\tilde{\kappa}}}$ and (4.9) to the event $\left\{\mu(\mathbf{0})=1\right.$ and $\left|\left[\partial C_{\kappa}\right]^{\mathbf{0}}\right|>N$ for all $\left.\kappa_{0} \leqq \kappa \leqq \tilde{\kappa}\right\} \quad$ (which implies $\left.\sum_{\mathbf{r} \in C_{\tilde{\kappa}}} \mu_{\Lambda}(\mathbf{r}) \geqq \frac{N}{2}\left(\tilde{\kappa}-\kappa_{0}\right)\right)$ we have

$$
\begin{aligned}
\varrho_{b}(\{\mu(\mathbf{0}) & \left.\left.=1 \text { and }\left|\left[\partial C_{\kappa}\right]^{\mathbf{0}}\right|>N \text { for all } \kappa_{0} \leqq \kappa \leqq \tilde{\kappa}\right\}\right) \\
& \leqq 2^{\left|\partial C_{\tilde{\kappa}}\right|} e^{-\beta b N\left(\tilde{\kappa}-\kappa_{0}\right) / 2} e^{4 \beta \tau\left|\partial C_{\tilde{\kappa}}\right|} \\
& \leqq\left(2 e^{4 \beta \tau}\right)^{8 \tilde{\kappa}} e^{-\beta b N\left(\tilde{\kappa}-\kappa_{0}\right) / 2}
\end{aligned}
$$

Given $\beta$, choose $N$ so that

$$
\beta b N / 2 \geqq 8(\log 2+4 \beta \tau) .
$$

Then by choosing first $\kappa_{0}$ large, then $\Lambda$ large (so $\tilde{\kappa}$ is large) we see from (4.12)-(4.13) that $\varrho_{b}(\{\mu(\mathbf{0})=1\})$ can be made as small as desired as $\Lambda \rightarrow \mathbb{Z}^{2}$, completing the proof of Theorem IV-4.

\section{Height Divergence Rate at High Temperature}

In this section we establish some additional results concerning the divergence rate of $E\left(h_{\Lambda, b}(\mathbf{0})\right)$ as $\Lambda \rightarrow \mathbb{Z}^{2}$ for $T>T_{c}(2)$. Our conclusion draws a clear distinction with 
the usual SOS model results where the divergence rate is logarithmic [FS; BEF], as will be seen. In the next proposition we denote by $R(\Lambda)$ the largest $\kappa$ such that the square $C_{\kappa}$ is contained in $\Lambda$.

Proposition V-1. For a given temperature $T$, if there exists $\gamma=\gamma(T)>0$ such that

$$
\operatorname{Pr}\left(h_{C_{\boldsymbol{R}}}(\mathbf{0})=0\right) \leqq e^{-\gamma R}
$$

for all $R$, then there exists $\gamma^{\prime}(\gamma)>0$ such that for any $b \geqq 0$,

$$
\operatorname{Pr}\left(h_{\Lambda, b}(\mathbf{0}) \geqq \gamma^{\prime} R(\Lambda) / \log R(\Lambda)\right) \rightarrow 1
$$

as $\Lambda \rightarrow \mathbb{Z}^{2}$.

Proof. We set $b=0$ in (5.2) since this case suffices by Proposition IV-1. Let $\mathscr{C}_{\Lambda}(\mathbf{r})$ denote the parallel spin cluster containing $\mathbf{r}$ obtained from $\left\{\sigma_{\Lambda}\left(\mathbf{r}^{\prime}\right): \mathbf{r}^{\prime} \in \mathbb{Z}^{2}\right\}$. Let

$$
A_{\Lambda}\left(\mathbf{r}, R^{\prime}\right)=\left\{\mathscr{C}_{\Lambda}(\mathbf{r}) \text { extends beyond } \mathbf{r}+C_{R^{\prime}}\right\} .
$$

Then we have

$$
\begin{aligned}
& \operatorname{Pr}\left(A_{\Lambda}\left(\mathbf{r}, R^{\prime}\right)\right)=\operatorname{Pr}\left(\left\{\sigma_{\Lambda}(\mathbf{r})=1\right\} \cup A_{\Lambda}\left(\mathbf{r}, R^{\prime}\right)\right)+\operatorname{Pr}\left(\left\{\sigma_{\Lambda}(\mathbf{r})=-1\right\} \cup A_{\Lambda}\left(\mathbf{r}, R^{\prime}\right)\right) \\
& \leqq 2 \operatorname{Pr}\left(\left\{\sigma_{\Lambda}(\mathbf{r})=1\right\} \cup A_{\Lambda}\left(\mathbf{r}, R^{\prime}\right)\right) \\
& \leqq 2 \operatorname{Pr}\left(\left\{\sigma_{\mathbf{r}+C_{R^{\prime}}}(\mathbf{r})=1\right\} \cup A_{\mathbf{r}+C_{R^{\prime}}}\left(\mathbf{r}, R^{\prime}\right)\right) \\
& =2 \operatorname{Pr}\left(h_{C_{R}}(\mathbf{0})=0\right) \text {, }
\end{aligned}
$$

by the FKG property of $\left\{\sigma_{\Lambda}(\mathbf{r})\right\}$ and its monotonicity in $\Lambda$, where we have assumed that $\mathbf{r} \in C_{R(\Lambda) / 2}$ and $R^{\prime} \leqq R(\Lambda) / 2$. Thus

$$
\operatorname{Pr}\left(A_{\Lambda}\left(\mathbf{r}, R^{\prime}\right)\right) \leqq 2 e^{-\gamma R^{\prime}}
$$

from the hypothesis of Proposition V-1. Now define distance between points in $\mathbb{Z}^{2}$ as

$$
\operatorname{dist}\left(\left(x_{1}, y_{1}\right),\left(x_{2}, y_{2}\right)\right)=\left|x_{1}-x_{2}\right|+\left|y_{1}-y_{2}\right|,
$$

and the diameter of subsets of $\mathbb{Z}^{2}$ as

$$
\operatorname{diam}(B)=\max _{\mathbf{r}_{1}, \mathbf{r}_{2} \in B} \operatorname{dist}\left(\mathbf{r}_{1}, \mathbf{r}_{2}\right) .
$$

Then for all $\mathbf{r} \in C_{R(\Lambda) / 2}$ and $R^{\prime} \leqq R(\Lambda) / 2$, we have

$$
\operatorname{Pr}\left(\operatorname{diam}(\mathscr{C}(\mathbf{r})) \geqq 2\left(2 R^{\prime}+1\right)\right) \leqq 2 e^{-\gamma R^{\prime}} .
$$

In order that $h_{\Lambda}(\mathbf{0}) \leqq k$, there must be a path from 0 beyond $\Lambda$ (and hence beyond $C_{R(1) / 2}$ ) which passes through $k$ or fewer distinct parallel spin clusters. Hence one of these clusters must have a diameter $\geqq R(\Lambda) / 2 k$. Now

$$
\operatorname{Pr}\left\{\max _{\mathrm{r} \in C_{R(\Lambda) / 2}}\left(\operatorname{diam} \mathscr{C}_{\Lambda}(\mathrm{r})\right) \geqq 2\left(2 R^{\prime}+1\right)\right\} \leqq 2(R(\Lambda)+1)^{2} e^{-\gamma R^{\prime}} .
$$

Thus

$$
\left.\operatorname{Pr}\left\{h_{\Lambda}(\mathbf{0}) \leqq k\right\} \leqq 2(R(\Lambda)+1)\right)^{2} e^{-\left(\frac{R(\Lambda)}{4 k}-1\right) / 2},
$$


and choosing $k=\gamma^{\prime} R(\Lambda) / \log R(\Lambda)$ gives

$$
\operatorname{Pr}\left\{h_{\Lambda}(0) \leqq \gamma^{\prime} R(\Lambda) / \log R(\Lambda)\right\}=O\left(R(\Lambda)^{2}\left(\frac{1}{R(\Lambda)}\right)^{\gamma /\left(8 \gamma^{\prime}\right)}\right)
$$

as $\Lambda \rightarrow \mathbb{Z}^{2}$. Proposition V-1 follows by choosing $\gamma^{\prime}<\gamma / 16$.

We now investigate the validity of the condition (5.1) which we used to establish Proposition V-1; it is presumably true for all $T>T_{c}(2)$, but for the moment all we can prove is that (5.1) is true if

$$
\left(1+e^{-8 \beta \tau}\right)^{-1}<p_{c}
$$

where $p_{c}$ is the critical probability for independent nearest neighbor site percolation on $\mathbb{Z}^{2}$. Since it is known rigorously that $p_{c}>1 / 2$ [T], (5.11) is valid for high temperature.

Proposition V-2. (5.1) is valid if $\beta$ satisfies (5.11).

Proof. The conditional probability that $\sigma(\mathbf{r})=1$ given $\left\{\sigma\left(\mathbf{r}^{\prime}\right): \mathbf{r}^{\prime} \neq \mathbf{r}\right\}$ is $e^{n \beta \tau} /(2 \cosh (n \beta \tau))$, where $n$ is the total spin of the sites adjacent to $\mathbf{r}$. This conditional probability is maximal when $n=4$ and is thus bounded by $1 /\left(1+e^{-8 \beta \tau}\right)$. Hence by a standard FKG argument

$$
\left\{\sigma_{\Lambda}(\mathbf{r}): \mathbf{r} \in \Lambda\right\} \prec\left\{\bar{\sigma}_{\Lambda}(r): \mathbf{r} \in \Lambda\right\},
$$

where the $\bar{\sigma}_{\Lambda}(\mathbf{r})$ are independent random variables with values \pm 1 and $\operatorname{Pr}\left(\bar{\sigma}_{\Lambda}(\mathbf{r})=1\right)=p$, where $p=1 /\left(1+e^{-8 \beta \tau}\right)$. This is so because the ratio of the joint densities of the unbarred and barred variables is a decreasing function of the spin configuration. The plus spin cluster at $\mathbf{r}$ for the barred variables, $\overline{\mathscr{C}}_{A}(\mathbf{r})$, is just an independent-site percolation cluster for percolation on $\Lambda \subset \mathbb{Z}^{2}$. It is known that for $p<p_{c}$

$$
\operatorname{Pr}\left\{\operatorname{diam}\left(\overline{\mathscr{C}}_{\mathbb{Z}^{2}}(\mathbf{0})\right) \geqq k\right\} \leqq e^{-\gamma^{\prime \prime}(p) k}
$$

with $\gamma^{\prime \prime}(p)>0$. (This follows from the results of [H] combined with those of $[\mathrm{M}]$ or [AB].) Hence by (5.12),

$$
\begin{aligned}
\operatorname{Pr}\left(h_{C_{R}}(\mathbf{0})=0\right) & \leqq \operatorname{Pr}\left\{\overline{\mathscr{C}}_{C_{R}}(\mathbf{0}) \text { reaches the boundary of } C_{R}\right\} \\
& =\operatorname{Pr}\left\{\overline{\mathscr{C}}_{\mathbb{Z}^{2}}(\mathbf{0}) \text { reaches the boundary of } C_{R}\right\} \\
& \leqq \operatorname{Pr}\left\{\operatorname{diam} \overline{\mathscr{C}}_{\mathbb{Z}^{2}}(\mathbf{0}) \geqq R\right\} \\
& \leqq e^{-\gamma^{\prime \prime}(p) R}
\end{aligned}
$$

by (5.13), which is what was needed.

\section{Appendix A: Inequalities}

We present here a number of results concerning monotonicity and correlation inequality properties of the height variables in our random surface model. For $\Lambda$ a finite (not necessarily connected) subset of $\mathbb{Z}^{2}$, let $\left\{\sigma_{\Lambda}(\mathbf{r}): \mathbf{r} \in \mathbb{Z}^{2}\right\}$ denote the \pm 1 valued spin variables of a standard $2-d$ Ising ferromagnet in zero magnetic field at temperature $T$ (fixed throughout this appendix) with plus boundary conditions 
outside of $\Lambda$ [so that $\sigma_{\Lambda}(\mathbf{r})=+1$ for each $\left.\mathbf{r} \notin \Lambda\right]$. Then the height variable $h_{\Lambda}(\mathbf{r})$ is defined as the minimum number of Peierls contours (or spin changes) crossed by any nearest neighbor path from $\mathbf{r}$ to $\Lambda^{c}$, the complement of $\Lambda$; for $\mathbf{r} \notin \Lambda, h_{\Lambda}(\mathbf{r})=0$.

The derivation of monotonicity and inequality properties of the $h_{\Lambda}(\mathbf{r})$ 's would be rather simple if they were monotone functions of the $\sigma_{\Lambda}(\mathbf{r})$ 's. However, simple examples show that the change of a spin from minus to plus can either raise or lower a height. Our derivation will thus be more involved. We next define

$$
\mu_{\Lambda}(\mathbf{r})= \begin{cases}1, & \text { if } h_{\Lambda}(\mathbf{r})=0 \\ 0, & \text { otherwise }\end{cases}
$$

The zero height region (where $\mu_{\Lambda}=1$ ) consists of those sites which are connected to $\Lambda^{c}$ by nearest neighbor paths passing only through plus spins; thus the $\mu_{\Lambda}(\mathbf{r})$ 's are monotone (non-decreasing) functions of the $\sigma_{\Lambda}(\mathbf{r})$ 's. Finally we define a random subset $L_{\Lambda}$ of $\Lambda$ as

$L_{\Lambda}=\left\{\mathbf{r} \in \Lambda: \mu_{\Lambda}(\mathbf{r})=0\right.$ and $\mathbf{r}$ is not a nearest neighbor of some

$$
\left.\mathbf{r}^{\prime} \in \mathbb{Z}^{2} \text { with } \mu_{\Lambda}\left(\mathbf{r}^{\prime}\right)=1\right\} \text {. }
$$

$L_{\Lambda}$ is decreasing in the $\sigma_{\Lambda}(\mathbf{r})$ 's; i.e., the occupation variables of $L_{\Lambda}$ are nonincreasing functions of the $\sigma_{\Lambda}(\mathbf{r})$ 's.

A key fact in understanding the structure of the random field $\left\{h_{\Lambda}(\mathbf{r})\right\}$ is that it can be constructed by a sequential procedure involving the zero height regions for a (random) sequence of nested subsets of the original $\Lambda$. In this construction, it is convenient to work with Ising models $\left\{\sigma_{\Lambda^{\prime}}(\mathbf{r})\right\}$ and corresponding $\left\{h_{\Lambda^{\prime}}(\mathbf{r})\right\},\left\{\mu_{\Lambda^{\prime}}(\mathbf{r})\right\}$ and $L_{\Lambda^{\prime}}$ simultaneously for all $\Lambda^{\prime} \subseteq \Lambda$ which are statistically independent of each other as $\Lambda^{\prime}$ varies. Starting from some particular configuration of the $\mu_{\Lambda}(\mathbf{r})$ 's and the corresponding $L_{\Lambda}$, we first note that $h_{\Lambda}(\mathbf{r})$ is determined for each $\mathbf{r}$ outside of $L_{\Lambda}: h_{\Lambda}(\mathbf{r})=0$ if $\mu_{\Lambda}(\mathbf{r})=1$ while $h_{\Lambda}(\mathbf{r})=1$ [and $\sigma_{\Lambda}(\mathbf{r})=-1$ ] for each $\mathbf{r}$ in $\Lambda \backslash L_{\Lambda}$ with $\mu_{\Lambda}(\mathbf{r})=0$. A key observation is that the conditional distribution [given $\left\{\mu_{\Lambda}(\mathbf{r})\right\}$ or $L_{\Lambda}$ ] of the $\sigma_{\Lambda}(\mathbf{r})$ 's for $r \in L_{\Lambda}$ is simply that of the standard Ising model with minus boundary conditions outside $L_{A}$; i.e., conditional on $L_{A}$,

$$
\left\{\sigma_{\Lambda}(\mathbf{r}): \mathbf{r} \in L_{\Lambda}\right\} \cong\left\{-\sigma_{L_{\Lambda}}(\mathbf{r}): \mathbf{r} \in L_{\Lambda}\right\},
$$

where $\cong$ denotes equidistribution. This implies that conditional on $L_{A}$,

$$
\left\{h_{\Lambda}(\mathbf{r}): \mathbf{r} \in L_{\Lambda}\right\} \cong\left\{1+h_{L_{\Lambda}}(\mathbf{r}): \mathbf{r} \in L_{\Lambda}\right\}
$$

We thus have the key formula,

$$
\left\{h_{\Lambda}(\mathbf{r}): \mathbf{r} \in \mathbb{Z}^{2}\right\} \cong\left\{\left(1-\mu_{\Lambda}(\mathbf{r})\right)\left(1+h_{L_{\Lambda}}(\mathbf{r})\right): \mathbf{r} \in \mathbb{Z}^{2}\right\} \text {. }
$$

Proposition A1. The random fields $\left\{h_{\Lambda}(\mathbf{r}): \mathbf{r} \in \mathbb{Z}^{2}\right\}$ are stochastically increasing in A; i.e.,

$$
E\left(f\left(\left\{h_{\Lambda^{\prime}}(\mathbf{r})\right\}\right)\right) \leqq E\left(f\left(\left\{h_{\Lambda}(\mathbf{r})\right\}\right)\right) \quad \text { for } \quad \Lambda^{\prime} \leqq \Lambda
$$

for any real-valued function $f$ depending on only finitely many height variables which is non-decreasing in each variable.

Proof. First we recall the standard fact that by the FKG inequalities for Ising spin variables $[\mathrm{FKG}],\left\{\sigma_{\Lambda}(\mathbf{r})\right\}$ (and hence also $\left.\left\{\mu_{\Lambda}(\mathbf{r})\right\}\right)$ is stochastically decreasing in $\Lambda$. 
This is so because the application of an infinite positive magnetic field to every site in $\Lambda \backslash \Lambda^{\prime}$ converts the distribution of $\left\{\sigma_{\Lambda}(\mathbf{r})\right\}$ to that of $\left\{\sigma_{\Lambda^{\prime}}(\mathbf{r})\right\}$.

We prove (A.6) by using (A.5) and induction on the number of sites in $\Lambda$. The case of a single site $\Lambda$ is trivial. For a larger $\Lambda$, we use (A.5) to write

$$
E\left(f\left(\left\{h_{\Lambda}(\mathbf{r})\right\}\right)\right)=E\left(g\left(\left\{\mu_{\Lambda}(\mathbf{r})\right\}\right)\right),
$$

where $g$ is the conditional expectation,

$$
g(\{\theta(\mathbf{r})\})=E\left(f\left(\left\{(1-\theta(\mathbf{r}))\left(1+h_{L_{\Lambda}}(\mathbf{r})\right)\right\}\right) \mid \mu_{\Lambda}(\mathbf{r})=\theta(\mathbf{r}) \text { for each } \mathbf{r}\right) .
$$

Now, suppose $\theta^{\prime}(\mathbf{r}) \geqq \theta(\mathbf{r})$ for each $\mathbf{r}$ and let $L^{\prime}, L$ be the corresponding $L_{\Lambda}$ 's (with $\left.L^{\prime} \cong L\right)$. Then

$$
\begin{aligned}
g(\{\theta(\mathbf{r})\}) & =E\left(f\left(\left\{(1-\theta(\mathbf{r}))\left(1+h_{L}(\mathbf{r})\right)\right\}\right)\right) \geqq E\left(f\left(\left\{\left(1-\theta^{\prime}(\mathbf{r})\right)\left(1+h_{L}(\mathbf{r})\right)\right\}\right)\right) \\
& \geqq E\left(f\left(\left\{\left(1-\theta^{\prime}(\mathbf{r})\right)\left(1+h_{L^{\prime}}(\mathbf{r})\right)\right\}\right)\right)=g\left(\left\{\theta^{\prime}(\mathbf{r})\right\}\right),
\end{aligned}
$$

where the first inequality follows from the monotonicity of $f$ and the second from the inductive hypothesis which is applicable since $L=L_{\boldsymbol{A}}$ has strictly fewer sites than $\Lambda$. Thus $g$ is a nonincreasing function of its arguments, and hence $E\left(g\left(\left\{\mu_{\Lambda}(\mathbf{r})\right\}\right)\right)$ is increasing in $\Lambda$ since $\left\{\mu_{\Lambda}(\mathbf{r})\right\}$ is stochastically decreasing in $\Lambda$. This completes the proof.

Proposition A2. The random field $\left\{h_{A}(\mathbf{r}): \mathbf{r} \in \mathbb{Z}^{2}\right\}$ has the $F K G$ property $[F K G]$ (or, equivalently, is called associated $[E P W]$ ); i.e., for any two real nondecreasing functions, $f$ and $g$, of finitely many variables,

$$
E\left(f\left(\left\{h_{\Lambda}(\mathbf{r})\right\}\right) g\left(\left\{h_{\Lambda}(\mathbf{r})\right\}\right)\right) \geqq E\left(f\left(\left\{h_{\Lambda}(\mathbf{r})\right\}\right)\right) E\left(g\left(\left\{h_{\Lambda}(\mathbf{r})\right\}\right)\right) .
$$

Proof. We again use the key formula (A.5). The spin random field $\left\{\sigma_{A}(\mathbf{r})\right\}$ has the FKG property [FKG] and hence so does $\left\{-\sigma_{\Lambda}(\mathbf{r})\right\}$. Since the $\left(1-\mu_{\Lambda}(\mathbf{r})\right)$ 's are nondecreasing functions of the $\left(-\sigma_{\Lambda}(\mathbf{r})\right)$ 's, they too have the FKG property. By induction on the number of sites in $\Lambda$, we may assume that conditional on $\left\{1-\mu_{\Lambda}(\mathbf{r})\right\},\left\{h_{L_{\Lambda}}(\mathbf{r})\right\}$ has the FKG property. Furthermore, by Proposition A1, the conditional distribution of $h_{L_{A}}(\mathbf{r})$ is stochastically increasing as a function of $\left\{\left(1-\mu_{\Lambda}(\mathbf{r})\right)\right\}$ (in the language of $[\mathrm{J}],\left\{h_{L_{\Lambda}}\right\}$ is a "monotone mixture" with $\left\{1-\mu_{\Lambda}\right\}$ ). It follows [J] that the double family of random variables $\left\{\left(1-\mu_{\Lambda}(\mathbf{r})\right), h_{L_{\Lambda}}(\mathbf{r})\right\}$ has the FKG property. Finally, formula (A.5) shows that the $h_{\Lambda}(\mathbf{r})$ 's are (equidistributed with) increasing functions of the double family and hence have the FKG property themselves.

As a standard application of monotonicity in $\Lambda$, given by Proposition A1, one has the existence of an infinite volume limit of the height variables, as follows.

Proposition A3. There exists a random field $\left\{h(\mathbf{r}): \mathbf{r} \in \mathbb{Z}^{2}\right\}$ taking values in the nonnegative integers or $+\infty$, such that

$$
\lim _{\Lambda \rightarrow \mathbb{Z}^{2}} E\left(f\left(\left\{h_{\Lambda}(\mathbf{r})\right\}\right)\right)=E(f(\{h(\mathbf{r})\})),
$$

where $f$ is any real function depending on finitely many variables which is either bounded or nondecreasing. The above limit may be taken through any increasing sequence of finite $\Lambda^{\prime}$ 's which eventually cover all of $\mathbb{Z}^{2}$. 
Proof. This is a consequence of the fact that the joint cumulative distribution function of finitely many $h_{\Lambda}(\mathbf{r})$ 's is monotonic in $\Lambda$.

Remark. In Sect. III of this paper, we show that, as one would expect, $h(\mathbf{r})$ can be identified with the minimum number of Peierls contours crossed to reach infinity from $\mathbf{r}$ in the infinite volume Ising model, $\{\sigma(\mathbf{r})\}=\lim _{\Lambda \rightarrow \mathbb{Z}^{2}}\left\{\sigma_{\Lambda}(\mathbf{r})\right\}$.

If one continues the sequential procedure, whose first step led to the key formula (A.5), in the obvious way, one obtains

$$
\left\{h_{A}\right\} \cong\left\{\left(1-\mu_{A}\right)+\left(1-\mu_{A}\right)\left(1-\mu_{L_{A}}\right)+\left(1-\mu_{A}\right)\left(1-\mu_{L_{A}}\right)\left(1-\mu_{L_{L_{A}}}\right)+\ldots\right\},
$$

where we have suppressed the $\mathbf{r}$ dependence. The sum on the right-hand side is really a finite series (for $\Lambda$ finite) because each $L_{\Lambda^{\prime}}$ is strictly contained in $\Lambda^{\prime}$. The next proposition weakens (A.12) to an inequality; we omit the proof which is similar to that of Propositions A1 and A2. We write $\left\{\phi_{1}(\mathbf{r})\right\} \prec\left\{\phi_{2}(\mathbf{r})\right\}$ when

$$
E\left(f\left(\left\{\phi_{1}(\mathbf{r})\right\}\right)\right) \leqq E\left(f\left(\left\{\phi_{2}(\mathbf{r})\right\}\right)\right),
$$

for every real nondecreasing function $f$ of finitely many variables.

Proposition A4. For any finite $\Lambda$, let $\left\{\mu_{\Lambda}^{(i)}(\mathbf{r})\right\}$, for $i=1,2, \ldots$, be an independent sequence of random fields, equidistributed with $\left\{\mu_{\Lambda}(\mathbf{r})\right\}$. Then

In particular, for $k=1,2, \ldots$,

$$
\left\{h_{\Lambda}\right\} \prec\left\{\sum_{j=1}^{\infty}\left[\left(1-\mu_{\Lambda}^{(1)}\right) \ldots\left(1-\mu_{\Lambda}^{(j)}\right)\right]\right\} .
$$

and

$$
\begin{gathered}
\operatorname{Pr}\left(h_{\Lambda}(\mathbf{r}) \geqq k\right) \leqq\left(1-\operatorname{Pr}\left(\mu_{\Lambda}(\mathbf{r})=1\right)\right)^{k}, \\
E\left(h_{\Lambda}(\mathbf{r})\right) \leqq \frac{1-\operatorname{Pr}\left(\mu_{\Lambda}(\mathbf{r})=1\right)}{\operatorname{Pr}\left(\mu_{\Lambda}(\mathbf{r})=1\right)} .
\end{gathered}
$$

Remark. The higher moments of $h_{\Lambda}(\mathbf{r})$ can be bounded, according to (A.14), by the moments of a geometric random variable with parameter $p_{\mathbf{r}}=\operatorname{Pr}\left(\mu_{\Lambda}(\mathbf{r})=1\right)$. A simpler explicit formula is obtained by bounding that geometric random variable by an appropriate exponential random variable:

$$
E\left(\left(h_{\Lambda}(\mathbf{r})\right)^{j}\right) \leqq \mid \log \left(1-p_{\mathbf{r}}\right)^{-j} \cdot j ! \leqq\left(p_{\mathbf{r}}\right)^{-j} \cdot j ! .
$$

Remark. The same reasoning which yielded (A.5) was used by Coniglio et al. [CNPR] to argue that

$$
\begin{aligned}
& E\left(\sigma_{\Lambda}(\mathbf{r})\right)=\operatorname{Pr}\left(\mu_{\Lambda}(\mathbf{r})=1\right)-\operatorname{Pr}\left(\mu_{\Lambda}(\mathbf{r})=0 \text { and } \mathbf{r} \notin L_{\Lambda}\right)-E\left(\sigma_{L_{\Lambda}}(\mathbf{r}) I\left(\mathbf{r} \in L_{\Lambda}\right)\right) \\
& \leqq \\
& \operatorname{Pr}\left(\mu_{\Lambda}(\mathbf{r})=1\right),
\end{aligned}
$$

so that (A.14-A.15) remain valid with $\operatorname{Pr}\left(\mu_{\Lambda}(\mathbf{r})=1\right)$ replaced by the finite volume magnetization, $E\left(\sigma_{\Lambda}(\mathbf{r})\right)$.

\section{Appendix B: The Multilayer Construction}

We construct a multilayer of molecules adsorbed on a substrate as follows: After Kossel [K] and Stransky [S], each molecule is represented by a unit cube whose 
corners are in $\mathbb{Z}^{3}=\mathbb{Z}^{3}+(1 / 2,1 / 2,0)$. The center of any such cube is restricted to be in $\overline{\mathbb{Z}}^{3 *}(+)=\left\{(x, y, z+1 / 2):(x, y, z) \in \mathbb{Z}^{3}, z \geqq 0\right\}$ so that the plane $\{z=0\}$ is the substrate surface.

Beginning with the bare substrate surface, we lay down unit cubes with centers at height $z=1 / 2$ to form a collection of rafts. Each raft is connected; by this we mean that the centers of any pair of cubes in the raft can be joined by an edge path in $\mathbb{Z}^{3 *}(+)$ lying entirely within the self-same raft. Any configuration with a collection of vacancies in any raft entirely surrounded by molecules is forbidden. As an example of this, consider 4 molecules with centers at $(1,0,1 / 2),(0,1,1 / 2)$, $(-1,0,1 / 2)$ and $(0,-1,1 / 2)$ : these surround the cube center $(0,0,1 / 2)$ which must therefore be occupied by a molecule.

Each raft is bounded by a closed connected strip of plaquettes in $\overline{\mathbf{Z}}^{3}$, each with normal parallel to the $\{z=0\}$ plane. The projection of each such strip down the (lll $\left.\begin{array}{lll}0 & 1\end{array}\right)$ axis onto the $\{z=0\}$ plane is a self-avoiding simple closed walk in $\mathbb{Z}^{2 *}$, the usual dual square lattice. The collection of such walks which describes a monolayer configuration is restricted to be edge-disjoint; this avoids doublecounting certain configurations, as can easily be checked. Different closed walks are however allowed to touch at vertices of $\mathbb{Z}^{2 *}$.

A second layer of adsorbed molecules is introduced by placing rafts of exactly the type described above on top of those already in place; no vertical overhangs are permitted. By following the projection procedure described above, this adds selfavoiding closed loops to those already constructed. We now require that all the closed loops obtained shall be edge-disjoint. This implies that we cannot place a molecule in the second layer vertically above one which happens to be in the edge of a raft in the first layer.

This process can be repeated, bearing in mind the restrictions introduced, to form multilayer configurations: these are isomorphic to the elements of the set of all edge-disjoint closed loops on $\mathbb{Z}^{2 *}$.

Let us now consider multilayer configurations constructed on a finite substrate plane, say $\{(x, y, 0):-N \leqq x \leqq N,-M \leqq y \leqq M\}$ with the associated loop representation on $\left\{(x, y) \in \mathbb{Z}^{2 *}:-(N+1)<x<(N+1),-(M+1)<y<(M+1)\right\}$ which is actually the Peierls contour, or low-temperature, expansion on $\mathbb{Z}^{2 *}$ for the planar spin-1/2 Ising model on the lattice

$$
\left\{(x, y) \in \mathbb{Z}^{2}:-N \leqq x \leqq N,-M \leqq y \leqq M\right\}=\Lambda_{N, M}
$$

with all the boundary spins fixed to point up. An example is shown in Fig. 1.

Evidently flat portions of the phase-separating surface forming the upper boundary of the multilayer configuration are represented by clusters of parallel spins: two spins, $\sigma(\mathbf{r})$ and $\sigma(\mathbf{s})$, for $\mathbf{r}, \mathbf{s} \in \Lambda_{N, M}$ belong to a + cluster (respectively cluster) means $\sigma(\mathbf{r})=\sigma(\mathbf{s})=+1$ (respectively -1 ) and there is a path connecting $\mathbf{r}$ and $\mathbf{s}$ of unit edges on $\Lambda_{N, M}$ on which all spins have value +1 (respectively -1 ). The surface height associated with any point $(x, y)$ in $\Lambda_{N, M}$ is the minimum number of Peierls contours on $\mathbb{Z}^{2 *}$ crossed, over all paths on $\Lambda_{N, M}$ from $(x, y)$ to the boundary $\partial \Lambda_{N, M}$.

We now discuss the energetics of the configurations generated in our model. The phase-separating surface $S$ is composed of elementary exposed faces of unit cubes which contribute surface-tension terms giving an energy

$$
\tau \sum_{\gamma \in \Gamma} l(\gamma)+\tau A_{1}
$$



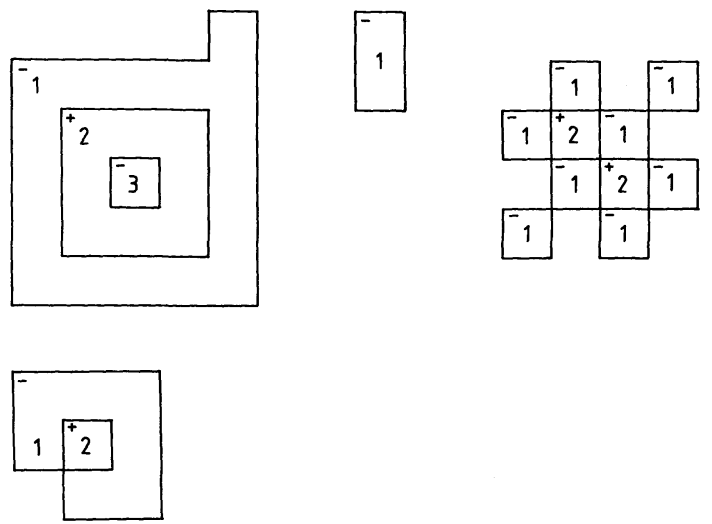

Fig. 1. A typical multilayer structure in the plane having four disjoint growths above the substrate plane. The two growths on the left are towers, the central one is a dimer, and the rightmost one corresponds to an antiferromagnetic patch. The numbers indicate the heights and the signs indicate the spin values of the different regions; the background has plus spin and zero height

where $\Gamma$ is the set of Peierls contours generated by $S$. The first term comes from faces perpendicular to the substrate plane; $l(\gamma)$ is the length of the simple closed walk $\gamma$ which is an element of the contour configuration $\Gamma$ given by the phaseseparating surface. The second term comes from all upper faces parallel to the substrate plane; $A_{1}$ is the number of such faces, which is obviously the same as the area of contact with the substrate. The interaction with the substrate is assumed to be

$$
-\varepsilon_{1} A_{1}
$$

giving a total energy,

$$
\mathscr{H}=\mathscr{H}(\Gamma)=\tau \sum_{\gamma \in \Gamma} l(\gamma)+\left(\tau-\varepsilon_{1}\right) A_{1} .
$$

For energetic stability against detachment from the substrate we require $\varepsilon_{1}>-\tau$ since the zero-temperature detached solid has two free planar surfaces with energy $2 \tau$ per unit area.

When $\tau=\varepsilon_{1}$, we recapture the zero-field Ising model with + boundary conditions. The extra term in (B.3) describes an external field $b=\tau-\varepsilon_{1}$, applied to the plus cluster attached to the boundary.

Acknowledgements. The authors thank J. Bricmont, M. Gelfand, and N. M. Švrakić very much for stimulating remarks. D. B.A. thanks the University of Arizona, Los Alamos National Laboratory and Clarkson University very much for hospitality and financial support while parts of this work were done.

\section{References}

[A] Abraham, D. B.: In Phase transitions and critical phenomena, Vol. 10. Domb, C., Lebowitz, J. L. (eds.). London: Academic Press 1986

[AB] Aizenman, M., Barsky, D. J.: Commun. Math. Phys. 108, 489 (1987) 
[AM] Abraham, D. B., Martin-Löf, A.: Commun. Math. Phys. 49, 35 (1976)

[AN] Abraham, D. B., Newman, C. M.: Phys. Rev. Lett. 61, 1969 (1988)

[AR] Abraham, D. B., Reed, P.: J. Phys. A 10, L121 (1977)

[B1] van Beijeren, H.: Commun. Math. Phys. 40, 1 (1975)

[B2] van Beijeren, H.: Phys. Rev. Lett. 38, 993 (1977)

[BCF] Burton, W. K., Cabrera, N., Frank, F. C.: Philos. Trans. Roy. Soc. (London) A 243, 299 (1951)

[BEF] Bricmont, J., El Mellouki, A., Fröhlich, J.: J. Stat. Phys. 42, 743 (1986)

[BGJS] Benettin, G., Gallavotti, G., Jona-Lasinio, G., Stella, A.: Commun. Math. Phys. 30, 45 (1973)

[BN] van Beijeren, H., Nolden, I.: The roughening transition. In Structure and dynamics of interfaces II, vol. 43 of current topics in physics. Berlin, Heidelberg, New York: Springer

[CNPR] Coniglio, A., Nappi, C. R., Peruggi, F., Russo, L.: Commun. Math. Phys. 51, 315 (1976)

[D] Dietrich, S.: In: Phase transitions and critical phenomena, vol. 12. Domb, C., Lebowitz, J. L. (eds.) London: Academic Press 1988

[Do] Dobrushin, R. L.: Th. Prob. Appl. 17, 582 (1972); 18, 253 (1973)

[EPW] Esary, J. D., Proschan, F., Walkup, D. W.: Ann. Math. Stat. 38, 1466 (1967)

[F] Forrester, P. J.: J. Phys. A 19, L143 (1986)

[FF] Fisher, D. S., Fisher, M. E.: Phys. Rev. 25, 3192 (1982)

[FKG] Fortuin, C. M., Kasteleyn, P. W., Ginibre, J.: Commun. Math. Phys. 22, 89 (1971)

[FS] Fröhlich, J., Spencer, T.: Phys. Rev. Lett. 46, 4006 (1981); Commun. Math. Phys. 81, 527 (1981); In: Scaling and self-similarity in physics. Fröhlich, J. (ed.). Basel: Birkhäuser 1983

[GKR] Gandolfi, A., Keane, M., Russo, L.: Ann. Probab. 16, 1147 (1988)

[H] Hammersley, J. M.: Ann. Math. Stat. 28, 790 (1957)

[J] Jogdeo, K.: Ann. Stat. 6, 232 (1978)

[K] Kossel, W.: Nachr. Ges. Wiss. Göttingen, Mathemat./Physikal. Klasse S. 135 (1927)

[L] Lieb, E. H.: Phys. Rev. Lett. 18, 1046 (1967)

[LW] Lieb, E. H., Wu, F. Y.: In: Phase transitions and critical phenomena, vol. 1. Domb, C., Green, M. S. (eds.). London: Academic Press 1972

[M] Menshikov, M. V.: Sov. Math. Dokl. 33, 856 (1986)

[O] Onsager, L.: Phys. Rev. 65, 117 (1944)

[R] Russo, L.: Commun. Math. Phys. 67, 251 (1979)

[S] Stransky, J. N.: Z. Phys. Chemie 136, 259 (1928)

[T] Toth, B.: Z. für Wahrscheinlichkeitstheorie 69, 19 (1985)

[W] Weeks, J. D.: In: Ordering in strongly fluctuating condensed matter systems. Riste, T. (ed.). New York: Plenum Press 1983

[Y] Yang, C. N.: Phys. Rev. 85, 809 (1952)

Communicated by A. Jaffe

Received April 29, 1989 